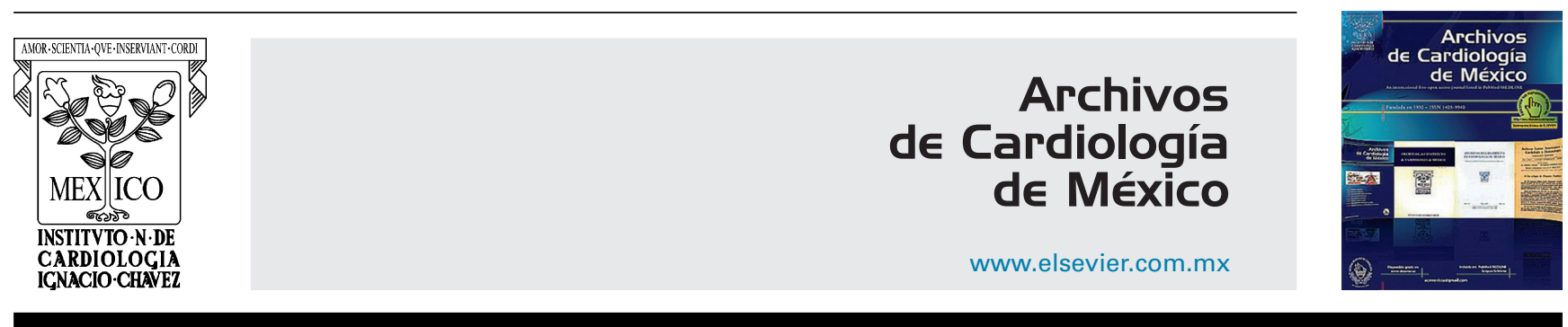

IMAGEN EN CARDIOLOGÍA

\title{
Aneurisma gigante de arteria coronaria descendente anterior
}

\section{Giant aneurysm of the left anterior descending coronary artery}

\author{
María Elena Arnáiz-García ${ }^{a, *}$, José Aurelio Sarralde-Aguayo ${ }^{b}$, Javier Arnáiz ${ }^{c}$ \\ y Ana María Arnáiz-García ${ }^{d}$
}

\author{
a Servicio de Cirugía Cardiovascular, Hospital Clínico Universitario de Salamanca, Salamanca, España \\ b Servicio de Cirugía Cardiovascular, Hospital Universitario Marqués de Valdecilla, Santander, Cantabria, España \\ c Servicio de Radiodiagnóstico, Aspetar-Orthopaedic and Sports Medicine Hospital, Doha, Qatar \\ ' Servicio de Medicina Interna, Hospital Universitario Marqués de Valdecilla, Santander, Cantabria, España
}

Recibido el 12 de marzo de 2018; aceptado el 1 de mayo de 2018

Paciente varón de 66 años, con antecedentes de hipertensión arterial mal controlada e hipercolesterolemia, que es atendido en nuestro centro aquejado de un dolor agudo e intenso a nivel centrotorácico, y siendo posteriormente diagnosticado de un infarto de miocardio de localización anterior. Tras realizarse una coronariografía se observa enfermedad coronaria de 3 vasos, con lesiones significativas a nivel de la coronaria derecha, arteria circunfleja y arteria coronaria descendente anterior. Además, se observó la presencia de un aneurisma coronario inmediatamente posterior a la estenosis del $90 \%$ presente a nivel de la arteria coronaria descendente anterior (fig. 1A). A través de esternotomía media, y bajo circulación extracorpórea y clampaje aórtico se realizó un cuádruple pontaje coronario. Se llevó a cabo una exclusión del aneurisma coronario, y se realizó un bypass secuencia con arteria mamaria interna izquierda para revascularizar el territorio de la arteria descendente anterior a 2 niveles, proximal y distal al aneurisma (fig. 1B). Tras la

\footnotetext{
* Autor para correspondencia. Servicio de Cirugía Cardiaca. Hospital Clínico Universitario de Salamanca. Paseo de San Vicente 58-182. 37007, Salamanca, España. Tel.: 923291263;. fax: 923291263.

Correo electrónico: elearnaiz@hotmail.com (M.E. Arnáiz-García).
}

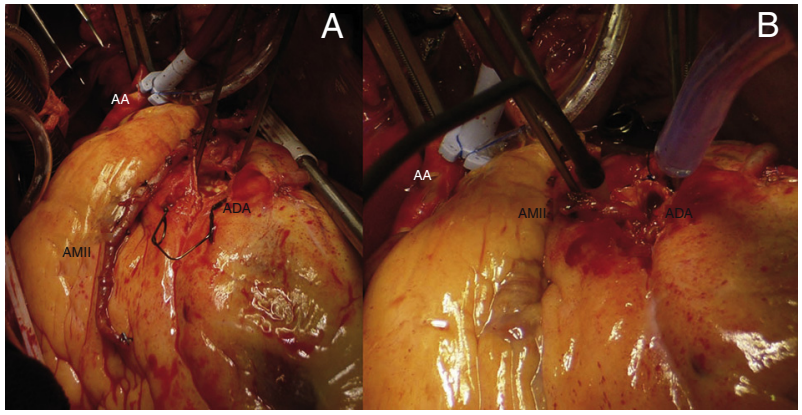

Figura 1 A) Imagen intraoperatoria del aneurisma de arteria coronaria descendente anterior y la arteria mamaria interna izquierda preparada para la realización del bypass coronario. B) Imagen postoperatoria mostrando la exclusión del aneurisma coronario con sutura, y la anastomosis distal del bypass coronario realizado.AA: aorta ascendente clampada; ADA: arteria coronaria descendente anterior; AMII: arteria mamaria interna izquierda.

cirugía, el paciente presentó una evolución satisfactoria, y pudo ser dado de alta al séptimo día del postoperatorio. Los aneurismas coronarios son infrecuentes y parecen presentar mecanismos etiopatogénicos similares a la enfermedad 
arterioesclerótica. Aquellos aneurismas de pequeño tamaño pueden ser tratados de forma conservadora o a través de procedimientos percutáneos. Sin embargo, en presencia de aneurismas coronarios gigantes, la cirugía parece ser el procedimiento indicado con el fin de evitar embolismos coronarios y complicaciones isquémicas secundarias ${ }^{1,2}$.

\section{Financiación}

No se recibió patrocinio de ningún tipo para llevar a cabo este artículo.

\section{Conflicto de intereses}

Los autores declaran no tener ningún conflicto de intereses.

\section{Bibliografía}

1. Flamariquea S, Cembreroa $\mathrm{H}$, Artaizc M, et al. Características morfológicas de los aneurismas de arterias coronarias. Incidencia e implicación clínica. Cir Cardiov. 2014;21:252-8.

2. Diayuan L, Qingyu W, Lizhong S, et al. Surgical treatment of giants artery aneurysm. J Thorac Cardiovasc Surg. 2005;130:817-21. 